\title{
Variability of extragalactic sources: its contribution to the link between ICRF and the future Gaia Celestial Reference Frame ${ }^{\star}$
}

\author{
F. Taris ${ }^{1}$, G. Damljanovic ${ }^{2}$, A. Andrei ${ }^{1,3,4}$, J. Souchay ${ }^{1}$, A. Klotz ${ }^{5,6}$, and F. Vachier ${ }^{7}$ \\ ${ }^{1}$ Observatoire de Paris - SYRTE, PSL Research University, CNRS/UMR 8630, Sorbonne Universités, \\ Université Pierre et Marie Curie, LNE, 61 avenue de l'Observatoire, 75014 Paris, France \\ e-mail: Francois.Taris@obspm.fr \\ 2 Astronomical Observatory, Volgina 7, 11060 Belgrade, Serbia \\ 3 Observatório Nacional/MCT, Rio de Janeiro, RJ 20921-400, Brazil \\ ${ }^{4}$ Obervatorio do Valongo, UFRJ, Rio de Janeiro, RJ 20080-090, Brazil \\ 5 Université de Toulouse, UPS/OMP, IRAP, 75014 Toulouse, France \\ ${ }^{6}$ CNRS, IRAP, 14 avenue Edouard Belin, 31400 Toulouse, France \\ 7 Observatoire de Paris - IMCCE, 75014 Paris, France
}

Received 13 June 2017 / Accepted 17 October 2017

\begin{abstract}
Context. The first release of the Gaia catalog is available since 14 September 2016. It is a first step in the realization of the future Gaia reference frame. This reference frame will be materialized by the optical positions of the sources and will be compared with and linked to the International Celestial Reference Frame, materialized by the radio position of extragalactic sources.

Aims. As in the radio domain, it can be reasonably postulated that quasar optical flux variations can alert us to potential changes in the source structure. These changes could have important implications for the position of the target photocenters (together with the evolution in time of these centers) and in parallel have consequences for the link of the reference systems.

Methods. A set of nine optical telescopes was used to monitor the magnitude variations, often at the same time as Gaia, thanks to the Gaia Observation Forecast Tool. The Allan variances, which are statistical tools widely used in the atomic time and frequency community, are introduced.

Results. This work describes the magnitude variations of 47 targets that are suitable for the link between reference systems. We also report on some implications for the Gaia catalog. For $95 \%$ of the observed targets, new information about their variability is reported. In the case of some targets that are well observed by the TAROT telescopes, the Allan time variance shows that the longest averaging period of the magnitudes is in the range 20-70 d. The observation period by Gaia for a single target largely exceeds these values, which might be a problem when the magnitude variations exhibit flicker or random walk noises. Preliminary computations show that if the coordinates of the targets studied in this paper were affected by a white-phase noise with a formal uncertainty of about 1 mas (due to astrophysical processes that are put in evidence by the magnitude variations of the sources), it would affect the precision of the link at the level of $50 \mu$ as.
\end{abstract}

Key words. reference systems - quasars: general - galaxies: photometry - methods: data analysis

\section{Introduction}

The Gaia satellite (Gaia Collaboration 2016a) was launched in December 2013, and the five-year nominal operations phase started in July 2014, after a half-year period of commissioning and performance verification. The collected data will allow the determination of highly accurate positions, parallaxes, and proper motions for more than one billion sources brighter than magnitude 20.7 in the white-light photometric $G$-band of Gaia. The number of quasars in these objects is estimated to be 500000 . Several intermediate releases, with a cadence of about a year, have been scheduled. The first release, Gaia Data Release 1 (DR1), was presented about 1000 days after launch (Gaia Collaboration 2016b; Fabricius 2016; Lindegren 2016).

Mignard et al. (2016) performed a comparison between a Gaia auxiliary quasar solution and the International Reference Frame (ICRF2) catalog. They found that 2191 sources in the

* Full Table 3 is only available at the CDS via anonymous ftp to cdsarc.u-strasbg.fr (130.79.128.5) or via

http://cdsarc.u-strasbg.fr/viz-bin/qcat?J/A+A/611/A52 auxiliary quasar solution have good optical positions that match the ICRF2 sources with high probability. The overall agreement between the optical and the radio positions is excellent: the angular separation is lower than 1 milliarcsec (mas) for $44 \%$ of the sources and lower than 10 mas for $94 \%$ of the sources. For the defining sources, the corresponding numbers are $71 \%$ and $98 \%$. In the sources with good optical and radio astrometry, no indication of physical optical-radio offsets exceeding a few tens of mas has been found. Of the sources with angular separations above 10 mas, $6 \%$ have statistically significant optical-radio offsets. We remark in the following on important aspects.

First, Petrov \& Kovalev (2017) pointed out that the auxiliary quasar solution is not yet published in full and that only the positions of $2 \%$ of the objects were reported. The question of how results of the comparison against this auxiliary solution are representative of the main solution of one billion objects remains open. Second, Mignard et al. (2016) used the ICRF2 catalog (Fey et al. 2015). By 14 September, 2016, the date of Gaia DR1 release, the total number of sources with positions determined with absolute astrometry using Very Long Baseline Interferometry (VLBI) reached 11444 , a factor of 3.5 increase 
with respect to the ICRF2. Third, the analysis of Mignard et al. (2016) showed that sources exist that have significant radiooptical offsets. If a population of genuine radio-optical offset sources exists, these significant offsets are challenging to explain.

Kovalev et al. (2017) studied a sample of active galactic nuclei (AGN) that showed a well-defined jet in VLBI. They found a statistically significant excess of sources with VLBI-to-Gaia positional offset directions along the jet for a full range of offset values as well as an excess for the direction opposite to the jet when offset values are lower than 3 mas. They claimed that a strong extended parsec-scale optical jet structure in many AGNs is required to explain the observed VLBI-Gaia offsets along the jet direction. The mas level offsets in the opposite direction were explained by a non-point-like VLBI jet structure or the "coreshift" effect that is due to synchrotron opacity.

The optical positional stability of quasars is critical because it puts in evidence astrophysical processes that can displace the photocenter of the sources and introduce an offset with the radiocenter. This offset, and its associated time variations, could strongly affect the link between the reference systems. Petrov \& Kovalev (2017) revealed a population of 472 objects with significant radio-optical offsets (median position offset of 2.2 mas) that cannot be explained by the random noise in the data. As seen previously, Kovalev et al. (2017) explained these offsets by a strong extended parsec-scale optical jet structure (along the jet for any scale from $\sim 1$ mas and larger or in the opposite direction for scales below $\sim 1$ mas).

The optical emission also comes from much more stable regions (the accretion disk, the basis of the jets, the broad line region (BLR), events on the host galaxy, or irregularities of the dust torus). However, precisely because it comes from different sources (although it is dominated somewhere in the accretion disk), the integrated light can indeed change the position of the photocenter as it undergoes variability. In short, optical flux variability should/could indicate variation of the photocenter, without necessarily indicating variation in the source structure. At the same time, by definition, optical flux variability will cause a variation in the magnitude, and hence in the error of the (Gaia) astrometric measurement, which of course will imply a nominal variation of the photocenter.

In the following, we present an exhaustive photometric study of 47 AGNs to increase the total number of targets that are suitable to build a link to the reference frames. In the next section, the set of nine optical telescopes we used to observe the targets is presented. These targets are described in the third section. The fourth section is dedicated to a comparison between the magnitudes measured by ground-based facilities and the Gaia $G$ band. Efforts have been made for simultaneous observations from our telescopes and Gaia. The last section introduces a statistical tool, the Allan variances, to characterize the optical stability of the targets that might be used to achieve the link between the reference systems. The conclusion gives some recommendations in the framework of the Gaia mission.

\section{Network of telescopes}

A set of optical telescopes is currently in use to observe AGN that could be candidates for the link between reference systems. Medium-sized telescopes ( $2 \mathrm{~m}$ to $4 \mathrm{~m}$ ) are used to obtain high-resolution images to scrutinize the morphology of the targets and their time evolution. Analyses of these images are currently in progress and will be published elsewhere. In addition, small ground-based telescopes ( $0.25 \mathrm{~m}$ to $2 \mathrm{~m}$ class telescopes) are dedicated to the monitoring of the target magnitudes. These telescopes are often robotic, which is mandatory to obtain wellsampled (one observation per day) and long-time (some years) observational series. In the frame of this study, nine telescopes were used, two of which were the two TAROT (French acronym for Télescope à action rapide pour les objets transitoires) telescopes. The associated image reduction has been described elsewhere (Taris et al. 2013, 2016).

A third instrument, the Telescope Joan Oró (TJO), is a $0.8 \mathrm{~m}$ robotic telescope ${ }^{1}$ operated since 2007 by the Institut d'Estudis Espacials de Catalunya (IEEC) in the Observatori Astronòmic del Montsec (OAdM). The images used in the frame of this study were acquired before June 2016, so that a specific process has been developed to reduce the data. The first step is the reduction of the raw images to obtain the science images, the raw images being corrected for instrumental effects such as bias, flat field by filter, and bad pixels. This step is achieved by the use of PRISM $^{2}$, a commercial software. The second step consists of the astrometric and photometric reductions that are achieved by the Gaia-GBOT Astrometric Reduction Pipeline ${ }^{3}$ (Bouquillon et al. 2014). We note that all the magnitudes obtained by the TJO are not photometricaly calibrated by observations of standard stars. They are relative magnitudes computed through a least-squares adjustment of the instrumental magnitudes of all the known objects in the field of view at the moment of observation.

Six other telescopes were used to determine magnitudes in the frame of this work. These magnitudes were derived from $3000 \mathrm{CCD}$ images taken during the period July 2013-November 2015. Five of the telescopes are part of a Serbian-Bulgarian mini-network of telescopes (Damljanović et al. 2014). Some of their characteristics are described in Table 1. The Astronomical Station Vidojevica (ASV) is a Serbian new observational site, on the mountain of Vidojevica. The $1.4 \mathrm{~m}$ telescope was recently installed (May 2016) within the frame of the BELISSIMA (BELgrade Initiative for Space Science, Instrumentation and Modeling in Astrophysics) project $^{4}$.

The last of the six telescopes is the $150 \mathrm{~cm}$ Leopold Figl Observatorium für Astrophysik (LFOA) telescope ${ }^{5}$.

For these six last telescopes, the photometric reduction process is the same, all the images are calibrated by identification and flux determination of comparison stars with known magnitudes. To identify them in the field of view (FoV) of quasars and to calculate their input $B V R$ magnitudes, we used the Sloan Digital Sky Survey (SDSS) catalog (Abazajian et al. 2009; York et al. 2000). Only stars in the magnitude range $14.5<g, r, i<19.5$ were selected (Chonis \& Gaskell 2008) from the catalog to minimize systematic effects. Comparison stars with $B V R$ measurements for differential photometry of QSOs are rare in the literature, therefore we converted the $u, g, r, i, z$ magnitudes of all identified stars into $B V R$ magnitudes using the algorithms and curves of Chonis \& Gaskell (2008) since our observations were obtained in the Johnson-Cousins filter system. We note that the identified comparison stars are not all appropriate for the differential photometry of quasars. Additional selection criteria for the comparison stars were applied and are summarized below.

First, stars with magnitudes and colors similar to QSOs (when possible) were chosen to minimize the extinction, color,

\footnotetext{
See wWW. oadm. cat/en/home.htm

See www.prism-america.com

See www . gbot . obspm . fr/index . php?page=pipeline

See http://belissima.aob.rs

See http: //astro.univie.ac.at/en/foa/home
} 
Table 1. Characteristics of telescopes used.

\begin{tabular}{|c|c|c|}
\hline Telescope-observatory & $\lambda, \phi, h$ & Focal plane \\
\hline $\begin{array}{c}\text { LFOA (UW) } \\
\text { Ritchey-Chrétien } \\
1520 / 12500\end{array}$ & $48.1,15.9,880$ & $\begin{array}{c}\text { SBIG ST-10XME } \\
2184 \times 1472 \\
6.8 \times 6.8 \\
0.15 \\
3.8 \times 5.6 \\
\end{array}$ \\
\hline $\begin{array}{l}\text { TAROT (OCA) } \\
\text { Newton } \\
250 / 800\end{array}$ & $6.9,43.8,1270$ & $\begin{array}{c}\text { EEV } 42-40 \text { BI } \\
2048 \times 2048 \\
13 \times 13 \\
3.5 \\
120 \times 120\end{array}$ \\
\hline $\begin{array}{l}\text { TAROT (ESO) } \\
\text { Newton } \\
250 / 800\end{array}$ & $70.7,-29.3,2347$ & $\begin{array}{c}\text { EEV } 42-40 \text { BI } \\
2048 \times 2048 \\
13 \times 13 \\
3.5 \\
120 \times 120 \\
\end{array}$ \\
\hline $\begin{array}{l}\text { TJO (OAdM) } \\
\text { Cassegrain } \\
800 / 7680\end{array}$ & $0.7,42.1,1570$ & $\begin{array}{c}\text { EEV } 42-40 \mathrm{BV} \\
2048 \times 2048 \\
13.5 \times 13.5 \\
0.36 \\
12.3 \times 12.3 \\
\end{array}$ \\
\hline $\begin{array}{c}\text { ASV(AOB)/Milanković tel. } \\
\text { Ritchey-Chrétien } \\
\text { Nasmyth, bent Cassegrain } \\
1400 / 11420\end{array}$ & $21.5,43.1,1150$ & $\begin{array}{c}\text { Apogee Alta U42 } \\
2048 \times 2048 \\
13.5 \times 13.5 \\
0.24 \\
8.3 \times 8.3\end{array}$ \\
\hline $\begin{array}{c}\text { ASV(AOB)/Nedeljković tel. } \\
\text { Cassegrain } \\
600 / 6000\end{array}$ & $21.5,43.1,1140$ & $\begin{array}{c}\text { Apogee Alta U42 } \\
2048 \times 2048 \\
13.5 \times 13.5 \\
0.46 \\
15.8 \times 15.8\end{array}$ \\
\hline $\begin{array}{l}\text { Rozhen (BAS) } \\
\text { Ritchey-Chrétien } \\
\text { 2000/15774 }\end{array}$ & $24.7,41.7,1730$ & $\begin{array}{c}\text { VersArray } 1300 \mathrm{~B} \\
1340 \times 1300 \\
20 \times 20 \\
0.26 \\
5.6 \times 5.6 \\
\end{array}$ \\
\hline $\begin{array}{c}\text { Rozhen (BAS) } \\
\text { Cassegrain } \\
600 / 7500\end{array}$ & $24.7,41.7,1760$ & $\begin{array}{c}\text { FLI PL09000 } \\
3056 \times 3056 \\
12 \times 12 \\
0.33 \\
16.8 \times 16.8\end{array}$ \\
\hline $\begin{array}{c}\text { Belogradchik (AO) } \\
\text { Cassegrain } \\
600 / 7500\end{array}$ & $22.7,43.6,650$ & $\begin{array}{c}\text { FLI PL09000 } \\
3056 \times 3056 \\
12 \times 12 \\
0.33 \\
16.8 \times 16.8\end{array}$ \\
\hline
\end{tabular}

Notes. LFOA (UW: Universitat Wien), TAROT (OCA: Observatoire de la Côte d'Azur, ESO: European Southern Observatory), TJO (OAdM: Observatori Astronomic del Montsec), and telescopes belonging to the Serbian-Bulgarian network (AOB: Astronomical Observatory of Belgrade, ASV: Astronomical Station Vidojevica, BAS: Bulgarian Academy of Science, AO: Astronomical Observatory). In the first column, we list the names of the observational stations and telescopes. The geographic coordinates (east longitude (in degrees) $\lambda$, latitude (in degrees) $\phi$, and altitude (in meters) $h$ ) are given in the second column. In the third column, we list the CCD cameras, the number of pixels, the pixel sizes (in $\mu \mathrm{m}$ ), the pixel scales (in arcsec/pixel), and the field of view (FoV, in arcmin).

and saturation effects. Then the stars were chosen to be as close as possible to the QSOs to lower the possible systematic errors that arise from flat-fielding and to increase the chance that they might be observable with telescopes with small FoVs. Finally, at least three stars were selected in each field to increase the accuracy of the QSOs photometric measurements and to minimize large systematic errors in the case that one of them had them.

After we applied these selection criteria, the set of remaining stars in the FoV of a target was used to perform ensemble photometry (e.g., Honeycutt 1992). The target magnitude was measured (aperture photometry with radius 4.7 arcsec), as well as the magnitude of each of the remaining stars (presented in each field), relative to the mean magnitude of the set of stars. Only a few magnitudes (per star and per filter) were obtained, which is in line with observational epochs, and the average magnitude, with its standard error, was computed. These calculated stellar magnitudes were in good agreement with the input magnitudes (the corresponding $B V R$ magnitudes obtained by transformation of the SDSS magnitudes). This was checked using a rejection criterion, which was set to $3 \sigma$ value. This criterion is sensitive to systematic errors such as star variability and instrumental and local errors. Some stars were removed during some of these steps. When no stars were located around QSOs in the SDSS, the APASS catalog ${ }^{6}$ was used to take $(B, V)$ values and to calculate the $R$ magnitude through a suitable transformation.

\section{Observed sources}

Bourda et al. (2008) published a first list of 70 targets to achieve the link between the reference systems, that is, the ICRF and the future Gaia Celestial Reference Frame (GCRF). One of the conclusions of this paper was that identifying more high-quality VLBI radio sources suitable for the alignment of ICRF against the GCRF was mandatory. A first set of 70 targets was observed in the optical wavelength by Taris et al. (2013, 2016). In 2011, Bourda et al. published a second set of 47 targets to increase the total number of targets suitable for the link. Different types of AGN can be found among these 117 targets, radio-loud AGN, such as QSO or BL Lac, and radio-quiet AGN, such as Seyfert galaxies. This mix of different objects could be a problem at optical wavelengths because some of these objects vary in spatial extent, and this variation evolves with time. Table 2 lists some elements of the morphology and variability at optical wavelengths in synthesized form. These elements were retrieved at the Centre des données astronomiques de Strasbourg ${ }^{7}$ (CDS). The table shows that very little information is known about the morphology and photometry/variability of these targets. Our measurements therefore add some new information about their magnitude variability. Table 3 lists all observations we obtained between 2013 and 2016, converted into the Gaia $G$ magnitude using the polynomial expression provided by Jordi et al. (2010).

\section{Gaia data and comparison to ground-based observations}

Quasars are known to be highly variable sources throughout the electromagnetic spectrum. In the optical wavelengths, Gaia will observe an estimated number of roughly 500000 quasars (Mignard 2012). In the first release, only one magnitude is given, with no attempt to a related epoch observation.

Gaia has two fields of view that are separated by a basic angle of 106.5 degrees. Gaia rotates around itself with a period of six hours. The period of the precession spin axis is 63 days. As a result, a sequence of measurements consists of several transits separated by different time intervals (from $0.62 \mathrm{~h}$

\footnotetext{
6 See http://www . aavso.org/apass

7 See http://cdsweb.u-strasbg.fr
} 
Table 2. Known morphology and variability for the 47 targets studied in this work.

\begin{tabular}{cccc}
\hline \hline QSO name & Morphology & Variability & Reference \\
\hline $0049+003$ & Unresolved & - & (M) Falomo et al. (2014) \\
$0109+200$ & Resolved & - & (M) Kacprzak et al. (2011) \\
$0210+515$ & Resolved & - & (M) Nilsson et al. (2007) \\
$0651+428$ & Resolved & - & (M) Nilsson et al. (2003) \\
$0652+426$ & Resolved & - & (M) Nilsson et al. (2003) \\
$0950+326$ & - & Variable & (V) Ravi Joshi (2013) \\
$1034+574$ & Unresolved & - & (M) Nilsson et al. (2003) \\
$1535+231$ & Resolved & - & (M) Nilsson et al. (2003) \\
$1722+119$ & Unresolved & Variable & (M) Urry et al. (2000) \\
& & & (V) Wierzcholska et al. (2015) \\
$1741+595$ & Resolved & - & (M) Nilsson et al. (2003) \\
$1811+317$ & Resolved & - & (M) Nilsson et al. (2003) \\
$1838+575$ & Resolved & - & (M) Paturel et al. (2003) \\
$2247+381$ & Resolved & - & (M) Nilsson et al. (2003) \\
\hline
\end{tabular}

Notes. The (M) or $(\mathrm{V})$ before the references refer to publications about morphology and/or variability. The sources with unknown morphology/variability are not listed in this table.

Table 3. Examples of observations from 2013 to 2016 in Gaia G band.

\begin{tabular}{cccc}
\hline \hline $\begin{array}{c}\text { Observatory } \\
\text { and QSO name }\end{array}$ & Obs. date (julian date) & $G$-Mag & Uncertainty $(1 \sigma)$ \\
\hline OCA-0007+106 & 2456658.258291 & 13.9542 & 0.021 \\
OCA-0007+106 & 2456679.276060 & 14.4303 & 0.037 \\
$\ldots$ & $\ldots$ & $\ldots$ & $\ldots$ \\
TJO-0049+003 & 2457221.60452418 & 15.829 & 0.030 \\
TJO-0049+003 & 2457244.55459490 & 15.818 & 0.024 \\
$\ldots$ & $\ldots$ & $\ldots$ & $\ldots$ \\
VBRL-0049+003 & 2456542.48674 & 16.036 & 0.025 \\
VBRL-0049+003 & 2456543.58256 & 16.015 & 0.036 \\
\hline
\end{tabular}

Notes. The full table is available at the CDS.

to $6.00 \mathrm{~h}$ ). When one sequence of transits is achieved, the following sequence appears about one month later because of the rotation axis precession and the satellite orbital motion. Between 40 and 250 measurements will be collected for each object during the five-year mission, depending on the ecliptic latitude, with a predicted mean number of $n_{\mathrm{obs}}=80$ measurements (Eyer \& Mignard 2005; Gaia Collaboration 2016a). The Gaia time sampling and the CCD data acquisition scheme allow probing stellar variability on a wide range of timescales (Eyer \& Mignard, 2005; Varadi et al. 2009) or with a multiperiodic sinusoidal signal (Mary et al. 2006). This is also a very important point for the variability of quasars, which are very well known to have timescale variabilities ranging between hours and years.

The Core Unit 7 (CU7; variability processing) of the Gaia Data Processing and Analysis Consortium (DPAC) is responsible for the variability analysis of more than a billion celestial sources (ESA \& DPAC 2016). The photometry is available in the form of per-field-of-view $G$-band flux time-series provided by CU5 (photometric processing). For the DR1 release, sources were only processed when they were located within 38 degrees from the south ecliptic pole and had a minimum of $20 \mathrm{FoV}$ $G$-band observations. Only two calibration models were used. The first, for the general time series, is a multi-frequency harmonic model with a low-order polynomial trend (each frequency has associated harmonics). The second, for specific object studies (SOS), is only used for Cepheids and RR Lyrae studies. In the first Gaia Data Release, no data were published about variability detection, period search/time-series modeling and classification, except for 599 Cepheids and 2595 RR Lyrae (3194 time series).
AGN, and more particularly, quasars, are therefore considered as invariable objects in the GDR1.

The principles of the photometric calibration of the $G$ band have been reviewed by Carrasco et al. (2016). Jordi et al. (2010) provided the relationships among the colors involving Gaia magnitudes (white-light $G$, blue $G_{\mathrm{BP}}$, red $G_{\mathrm{RP}}$, and $G_{\mathrm{RVs}}$ bands) and colors from other commonly used photometric systems (particularly Johnson-Cousins). The following polynomial expression is of special interest in the framework of this study:

$$
\begin{array}{r}
G-V=-0.0120-0.3502\left(V-R_{\mathrm{C}}\right)-0.6105\left(V-R_{\mathrm{C}}\right)^{2}+\ldots \\
\ldots+0.0852\left(V-R_{\mathrm{C}}\right)^{3} .
\end{array}
$$

We emphasize that the previous relation was obtained through the analysis of the BaSeL3.1 stellar spectral energy distribution (SED) library, essentially for stars with $T_{\text {eff }}>4500 \mathrm{~K}$ (for $T_{\text {eff }}<4500 \mathrm{~K}$ dispersion exists). As a consequence, this relation is probably not well suited to convert the quasar magnitudes, obtained by ground-based telescopes with the $\left(V, R_{\mathrm{C}}\right)$ Johnson-Cousins filters, into the Gaia $G$ band. It is of importance to note that the color-color diagram is a powerful method to distinguish between stars and quasars (Palanque-Delabrouille et al. 2011; Wu et al. 2012) because most quasars (with redshifts lower than four) can be easily separated from both normal and late-type stars by their position (locus) in a color-color or colorcolor-color diagram (Newberg \& Yanni 1997).

The CU4 (object processing) is in charge of processing nonsingle stars, solar system objects, and extended objects (EO), the latter through the Developments Unit (DU) 470 (extended objects). Based on a flag given by the CU5, DU 470 will check for a sign of host galaxy structures around the quasar. Nevertheless, these particular EO were not treated for the Gaia DR1 release and are not listed as such in the catalog (ESA \& DPAC 2016). This problem is of importance for the determination of the magnitude since it has been emphasized previously that some objects have a large extension on the sky.

\section{Photometric curves of quasars}

Figures 1 and B.1 to B.6 also present a comparison of the GDR1 $G$-band magnitude against ground-based observations. As previously mentioned, in the GDR1, quasars are considered as point-like objects without a host galaxy. When they are extended objects and observed from the Earth, the magnitudes are always computed by the aperture photometry technique. Moreover, in the case of the DR1, quasars are considered as invariable objects, and magnitude measurements per transit have been averaged. The polynomial expression for the color-color transformation from $\left(V-R_{\mathrm{C}}\right)$ to $(G-V)$ given by Jordi et al. (2010) was used, with the restrictions mentioned above. In Figs. 1 to B.6, the Gaia $G$-band magnitude is represented by a horizontal black line during the time of observation by Gaia. The differences that can be seen between target magnitudes can be explained by the restrictions described above.

The Gaia Observation Forecast Tool ${ }^{8}$ is intended to help astronomers find out when their targets will be observed by Gaia. Observation times for 53 targets have been computed with this software from 26 September 2014 to 14 September 2016, the publication date of the GDR1. They appear as small vertical green lines at the bottom of each graph in Figs. 1 to B.6. These observation epochs are also used in the section about the Allan variances.

8 See www.cosmos.esa.int/web/gaia/tools 

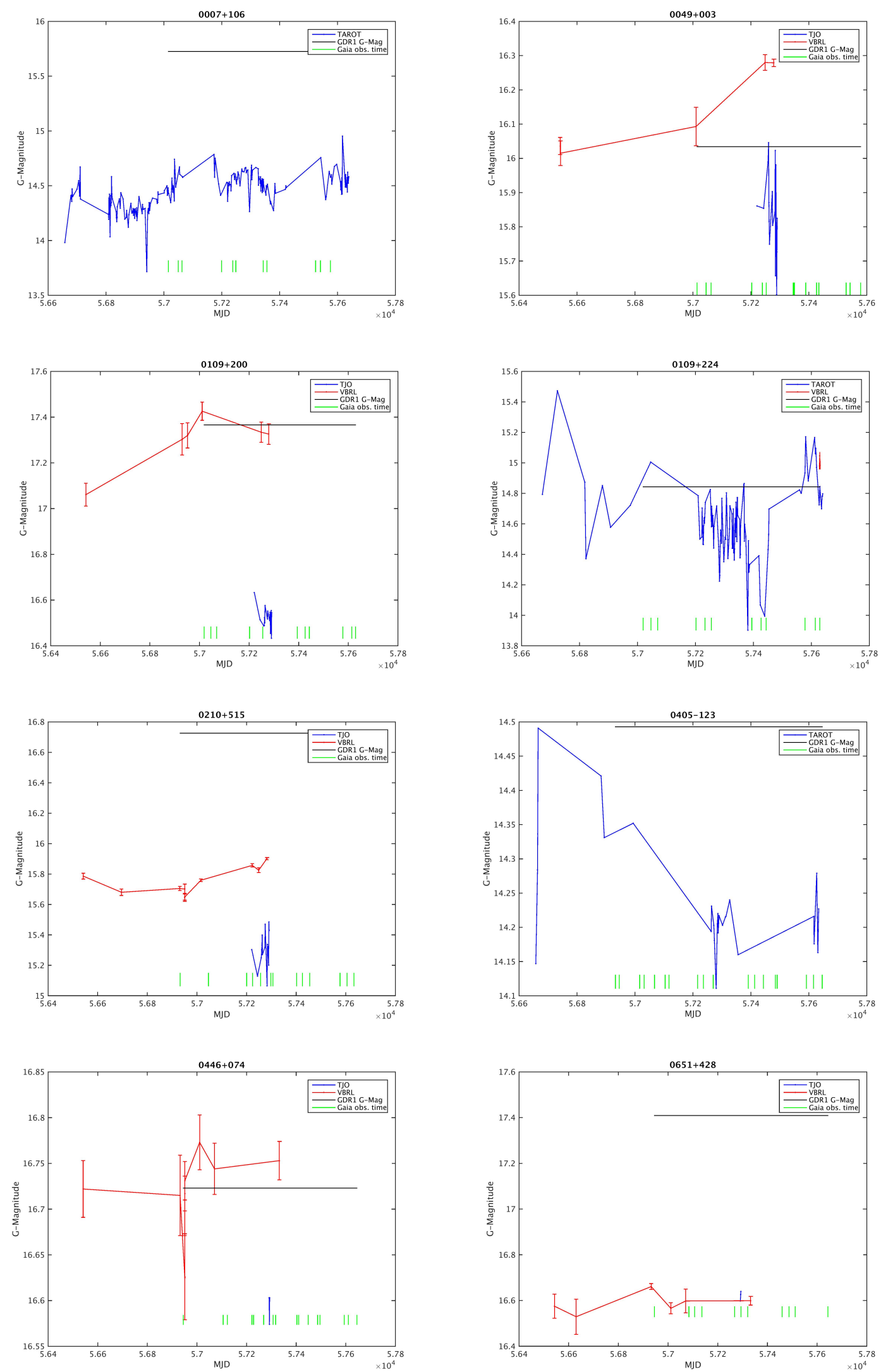

Fig. 1. Comparison of $G$ magnitudes for QSOs 0007+106, 0049+003, 0109+200, 0109+224, 0210+515, 0405-123, 0446+074, and 0651+428 from top to bottom and left to right. The vertical green ticks are the observation times by Gaia, the horizontal black line is the GDR1 magnitude. The other curves are the $G$ magnitudes observed by the Telescope Joan Oro (TJO), the TAROT telescopes (TAROT), and/or the VidojevicaBelogradchik-Rozhen-LFOA telescopes (VBRL), as indicated at the top right of each panel. 

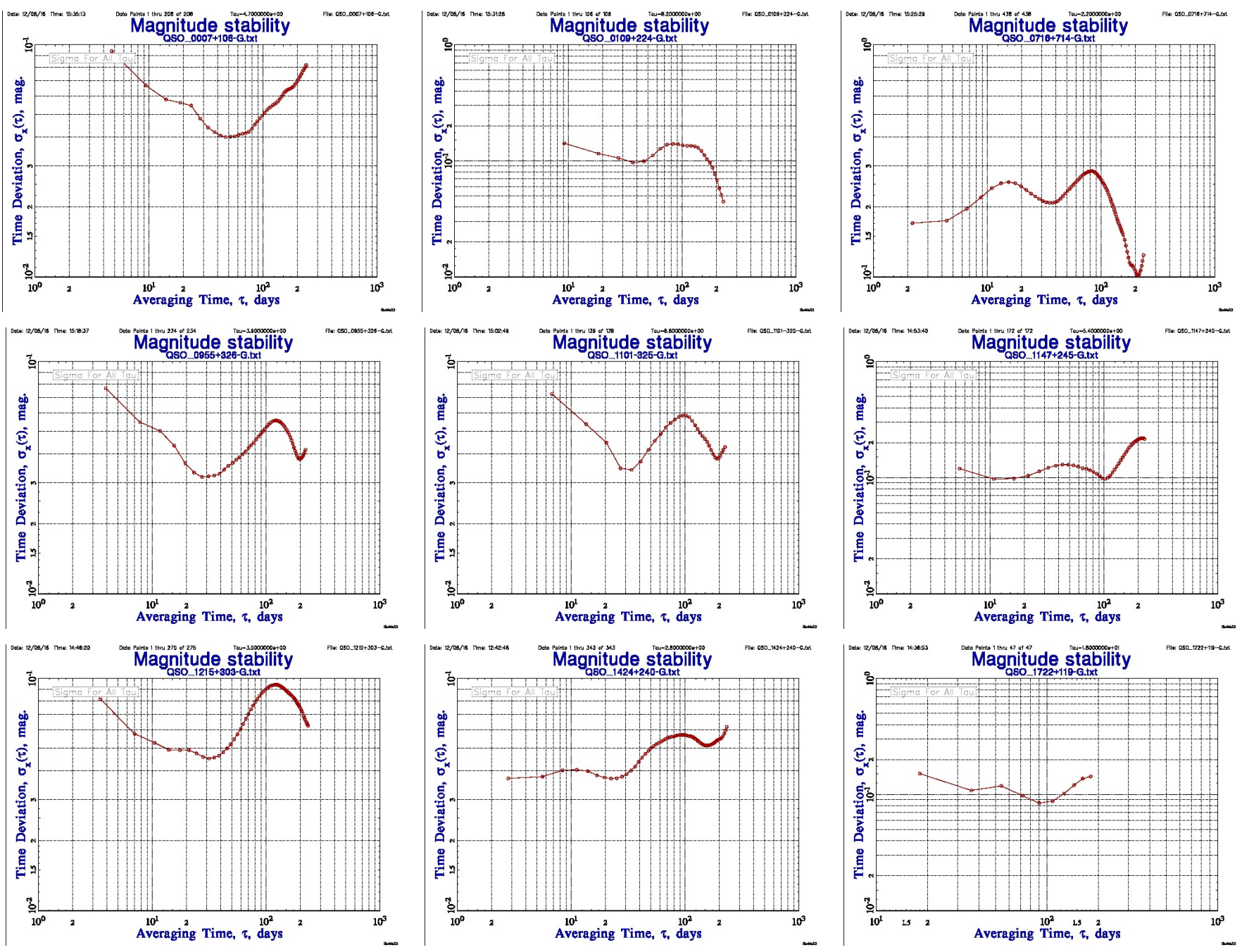

Fig. 2. Time deviation for QSOs $0007+106,0109+224,0716+714,0955+326,1101-325,1147+245,1215+303$, 1424+240, and $1722+119$ from top to bottom and left to right.

\section{Allan variances}

\subsection{Essential features}

The Allan variances are statistical tools that are very common and used in time and frequency metrology. We refer to Sullivan et al. (1990) and Riley (2008) for a very complete review of these tools. The essential features are given in the appendix. They must be known to fully understand the results. The atomic clocks and frequency standards are affected by five types of noises: white-phase noise (White PM), flickerphase noise (Flicker PM), random-walk-phase noise or whitefrequency noise (RW PM/White FM), flicker-frequency noise (Flicker FM), and random-walk-frequency noise (RW FM). In the same way, time series of quasar magnitude are also affected by white and random walk noises (Kelly et al. 2009). Allan variances can then be used to characterized the stability of quasar magnitude because the five-noise model of an atomic frequency standard is a generalization for quasar time-series.

\subsection{Characterization of the Allan time deviation of our set of AGNs}

The Allan time variance was applied to some magnitude timeseries presented in Sect. 4. Figure 2 shows the results for nine well-observed targets (long time-series with short sampling time) by the TAROT telescopes. The first eight targets are ICRF sources, but the ninth is not.

All the stability curves are affected by one (or more) periodic phenomenon. This result agrees with the conclusions obtained by Taris et al. (2016). Nevertheless, the Allan variances are probably poorly suited to distinguish periodic signals with different amplitudes. Only the predominant signal is emphasized by the Allan variances. All the frequency stability graphs show that periodic signal(s) prevent us from considering that the photometric curves are affected by white-phase noise (or at worst, flicker-phase noise) for averaging times longer than one or two months. This means that it is not possible to compute an average magnitude beyond such a time sampling simply because the underlying stochastic process is not stationary (random-walk-phase modulation). When it is possible, the epoch of observation, or equivalently an average epoch, must be given. TDEV is equal to the standard deviation of time variations (here: magnitudes variations) for white-phase modulation noise. Then the first point of a $\sigma / \tau$ graph provides direct access to the standard deviation of the magnitude measurements (the white-phase noise is the measurement noise). Table 4 lists for the nine previous targets the TDEV values for the average period of observation $\tau_{0}$. Based on the curves of Fig. 2, we also show an approximate of the longest averaging period $P_{\text {Max }}$. $P_{\text {Max }}$ must be viewed in relation with 
Table 4. TDEV and characteristic times for the targets of Fig. 2.

\begin{tabular}{cccc}
\hline \hline QSO name & $\tau_{0}($ days $)$ & $\sigma_{x}\left(\tau_{0}\right)(\mathrm{mag})$ & $P_{\text {Max }}($ days $)$ \\
\hline $0007+106$ & 4.7 & 0.094 & 70 \\
$0109+224$ & 9.2 & 0.142 & 45 \\
$0716+714$ & 2.2 & 0.170 & 50 \\
$0955+326$ & 3.9 & 0.077 & 30 \\
$1101-325$ & 6.8 & 0.073 & 35 \\
$1147+245$ & 5.4 & 0.119 & 20 \\
$1215+303$ & 3.5 & 0.081 & 40 \\
$1424+240$ & 2.8 & 0.037 & 30 \\
$1722+119$ & 18.0 & 0.151 & 35 \\
\hline
\end{tabular}

the observation epochs computed by the Gaia Observation Forecast Tool. When the targets are observed during periods shorter than $P_{\text {Max }}$, it is always possible to average the magnitudes, the standard deviation of the mean being $\sigma_{x}\left(\tau_{0}\right)$, with $\tau_{0}$ the average observation time (or the time between two observations). For observation times separated by more than $P_{\mathrm{Max}}$, the magnitudes cannot be averaged since the underlying stochastic process is not stationary, the consequence being that the computed average magnitude will be biased. In other words, for all the intermediate release, except perhaps for the last release, where all epoch and transit data will be included, the quasar magnitudes will probably be biased. A concrete example is given by the case of QSO $0716+714$, which has a mean magnitude of $13.18 \pm 0.03$ (TAROT observations, Fig. 1), which is similar to the Gaia $G$ band magnitude (13.277). Table 4 shows that $P_{\text {Max }}$ is 50 days. It is obvious from the graph that the average $G$ magnitude on such a time sampling can vary between 12.3 and 13.9 , that is to say, an amplitude variation of 1.6 magnitudes. It is then important, in all the intermediate releases of the Gaia catalog, to have access to the transit data to overcome bias that is due to the periodic phenomenon or to astrophysical processes inside quasars.

Table 4 shows that the most photometrically stable source is QSO $1424+240\left(\sigma_{x}\left(\tau_{0}\right)=0.037 \mathrm{mag}\right)$ and that the most unstable is QSO $0716+714\left(\sigma_{x}\left(\tau_{0}\right)=0.170 \mathrm{mag}\right)$. Some authors (Andrei et al. 2008; Taris et al. 2011, 2013, 2016; Popovic et al. 2012) pointed out that high photometric variations would make a given quasar less suitable to create a stable extragalactic reference frame. The TVAR tool allows quantifying the photometric quality of quasars and can help to select sources for the link between reference systems. This therefore is a way to propose other targets that might be suitable for this link.

In the radio domain $(2.3$ and $8.4 \mathrm{GHz})$, the appearance of new quasar components corresponds to an increase in quasar flux density, and the corresponding light curves indicate potential changes in source structure before they appear in VLBI images (Shabala et al. 2014). The relationship between the astrometric and photometric variability cannot be assumed as a general rule, although it may exist particularly in the $\mu$ as astrometric regime (Popovic et al, 2012). It will be of interest to compare astrometric and photometric Gaia time series with each other and with the TVAR variance. This is the object of the Test Case WP944-VAL060-005 of the CU9 (Validation Tests Specification). It might confirm the possible relationship describe above and also help to select good quasar candidates for the link between reference systems. Moreover, in the particular case of the GCRF, this relation could restrict the number of targets coming from the Gaia Initial QSO Catalog (GIQC), which contains 191372 defining targets.

\section{Consequences of quasar variability for the stability of the link between reference frames}

As we recalled in the introduction of this paper, the optical emission of quasars comes from different sources. Strong extended parsec-scale jet structure, as emphasized by Kovalev et al. (2017) and sources near the black hole (Lobanov \& Roland 2005), but also more stable regions: the accretion disk, the basis of the jet, the BLR, the host galaxy, or irregularities of the dust torus (Popovic et al. 2012; Li \& Cao 2008; Shalyapin et al. 2002; Bahcall et al. 1995; Cheung 2003). Even though it is an extrinsic phenomenon, the long-term optical variability in AGN could also be due to gravitational microlensing (Hawkins et al. 2003). At the moment, very little is known about the relation between quasar variability and the jitter in their photocenter. Taris et al. (2011, 2013, 2016), Popovic et al. (2012), and Kovalev et al. (2017) have reported numerical values for the photocenter jitter between some tens of $\mu$ as to some mas.

Mignard et al. (2016) have studied the frequency distribution of the formal uncertainties in right ascension and declination of the optical positions of ICRF2 sources given by Gaia. The median values are 0.62 mas and 0.56 mas, respectively, for the 2191 studied sources. These last values are in good agreement with what is expected for the photocentric jitter.

It is therefore of interest to study the consequences of quasar variability for the precision of the link between reference frames. The word "precision" is used in place of stability from here on; the precision is defined as the standard deviation of the mean. The 47 targets we studied for this paper are currently not part of the ICRF2 reference frame. The coordinates computed by Petrov (2011) were used. They were derived with millisecond accuracies from an analysis of VLBI observations using an absolute astrometry method. The accuracies of the source coordinates are in the range of $0.3-7$ mas, with a median of 1.1 mas.

We recalled at the beginning of Sect. 4 that the Gaia scanning law will produce 80 observations per object on average during the five years of the nominal mission. These 80 observations will not be regularly time-sampled during the Gaia mission. Nevertheless, to simplify the computational process, 80 white-phase noise measurements were added to the initial positions given by Petrov (2011); these measurements are considered as regularly spaced data. This built time series of the photocentric jitter that is due to the effect of astrophysical processes that are put in evidence by the magnitude variations of the sources. The formal uncertainties of the 47 white-phase noise time series were chosen to recover the frequency distribution obtained by Mignard et al. (2016). For example, $6 \%$ of the targets are affected by a white-phase noise with a formal uncertainty of 0.2 mas, $30 \%$ have a 0.4 mas uncertainty, etc. Figure 3 shows an example of the photocenter jitter for QSO 1838+575 ( $\sigma=1.2$ mas). A given formal uncertainty has been randomly assumed for a given target. All these formal uncertainties are listed in Table 5 so that the frequency distribution given by Mignard et al. (2016) can be recovered.

To study the precision of the link between reference frames, we followed the method used by Lambert (2013, 2017, pers. comm.). The coordinate transformation consists of three rotations, of angles noted $R_{i}$, plus another parameters $D_{i}$, expressing a dipolar deformation of the coordinate field. This corresponds to the toroidal and spheroidal harmonics of the vector spherical harmonics development of a vector field (Mignard \& Klioner 2012). Eighty values of these six parameters 


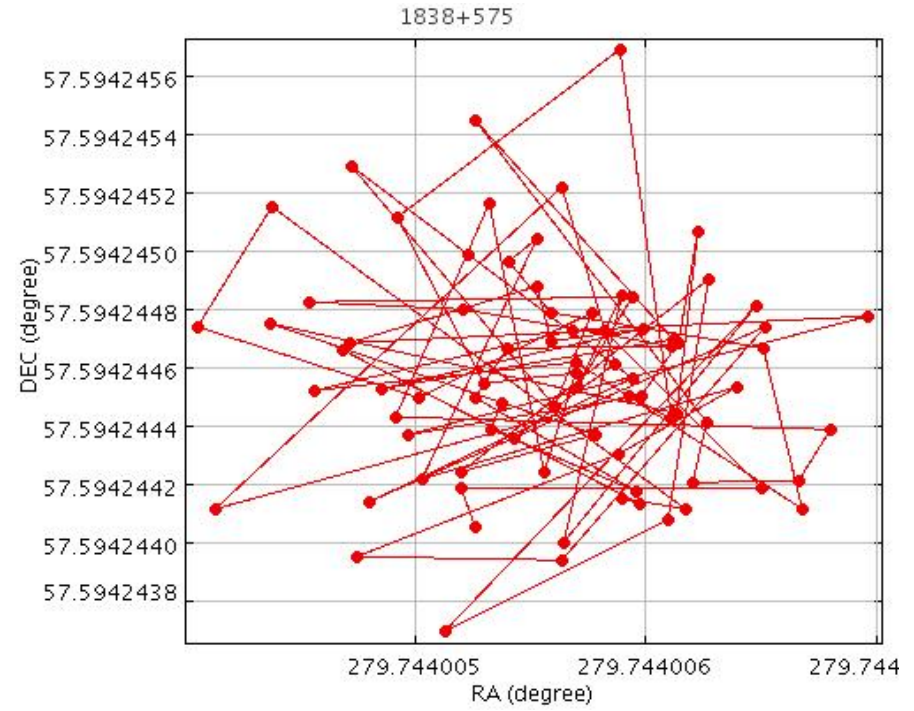

Fig. 3. Photocenter jitter for QSO $1838+575$. The photocenter is affected by white-phase noise with $\sigma=1.2$ mas. Eighty position measurements have been simulated.

are obtained by a least-squares adjustment through these two equations:

$$
\begin{aligned}
\Delta \alpha= & {\left[R_{1} \cos \alpha \tan \delta+R_{2} \sin \alpha \tan \delta-R_{3}\right] } \\
& -\left(D_{1} \sin \alpha-D_{2} \cos \alpha\right) / \cos \delta,
\end{aligned}
$$

$$
\begin{aligned}
\Delta \delta= & {\left[-R_{1} \sin \alpha+R_{2} \cos \alpha\right]-D_{1} \cos \alpha \sin \delta } \\
& -D_{2} \sin \alpha \sin \delta+D_{3} \cos \delta .
\end{aligned}
$$

In each of them, the first term in brackets corresponds to the rotation and the second term expresses the dipolar deformation. The results of this computation (mean values of the 80 parameters $R_{i}$ and $D_{i}$ together with the associated precision) are listed in Table 6. The first part of this table lists the statistics of the coordinate comparison. The mean difference in $\Delta \alpha \cos \delta$ and $\Delta \delta$ is $1 \mu$ as and $29 \mu$ as, respectively, the formal error being roughly 1 mas in the two cases. This means that the frequency distribution obtained by Mignard et al. (2016) could equivalently be replaced by a white-phase noise with a formal uncertainty of about 1mas. This would have the same effect on the rms of the parameters $R_{i}$ and $D_{i}$ (of course, not on their numerical values). The second part of Table 6 lists the numerical values of the rotation angles and glide. The precision of the rotation angles $R_{i}$ is between 30 and $70 \mu$ as (50 $\mu$ as on average). The glide is also important in this case because of the spatial distribution of the chosen targets. The targets all lie in the northern hemisphere, the precision of the parameters $D_{i}$ being between 40 and $60 \mu$ as (50 $\mu$ as on average).

The Gaia astrometric accuracy that could be obtained at the end of the mission is expected to be lower than $100 \mu$ as (Mignard 2013) for a target with a magnitude $G=18$; this magnitude is the limiting magnitude of the targets that are suitable to achieve the link between the ICRF and the future GCRF (Bourda et al. 2008). No reference to a relation between the quasar variability and their photocenter jitter is made in this case. With this formal uncertainty about the position of quasars, the precision of the link for all the parameters $R_{i}$ and $D_{i}$ is $4 \mu$ as on average. This last numerical value can be directly compared to the precision of the parameters obtained when the variability of quasars is accounted for.

The Gaia frame will be formed by roughly 10000 quasars, presumably the steadiest quasars. About a few hundred of them
Table 5. Formal uncertainties (mas) of the white-phase noise for each QSO of this study.

\begin{tabular}{ccc}
\hline \hline QSO name & $\sigma(\Delta \alpha \cos \delta)$ & $\sigma(\Delta \delta)$ \\
\hline $0049+003$ & 0.396 & 0.411 \\
$0109+200$ & 1.590 & 1.484 \\
$0210+515$ & 1.633 & 1.949 \\
$0446+074$ & 0.911 & 0.902 \\
$0651+428$ & 1.491 & 1.253 \\
$0652+426$ & 0.421 & 0.418 \\
$0741+294$ & 1.922 & 2.268 \\
$0838+235$ & 0.566 & 0.626 \\
$0838+456$ & 0.645 & 0.639 \\
$0850+284$ & 0.543 & 0.653 \\
$0854+334$ & 2.131 & 2.190 \\
$0907+336$ & 0.419 & 0.370 \\
$0950+326$ & 0.865 & 0.765 \\
$0952+338$ & 0.556 & 0.649 \\
$1020+292$ & 0.836 & 0.872 \\
$1032+354$ & 0.396 & 0.418 \\
$1034+574$ & 0.177 & 0.206 \\
$1145+321$ & 0.623 & 0.621 \\
$1201+454$ & 0.852 & 0.876 \\
$1212+467$ & 0.393 & 0.413 \\
$1228+077$ & 0.855 & 0.793 \\
$1242+574$ & 0.322 & 0.432 \\
$1312+240$ & 0.411 & 0.374 \\
$1345+735$ & 0.931 & 0.804 \\
$1429+249$ & 0.356 & 0.356 \\
$1518+162$ & 0.731 & 0.812 \\
$1535+231$ & 0.210 & 0.186 \\
$1556+335$ & 1.391 & 1.440 \\
$1603+699$ & 0.565 & 0.564 \\
$1607+604$ & 0.372 & 0.411 \\
$1612+378$ & 1.212 & 1.468 \\
$1618+530$ & 0.566 & 0.584 \\
$1722+119$ & 0.367 & 0.395 \\
$1730+604$ & 0.972 & 1.018 \\
$1741+595$ & 1.014 & 0.979 \\
$1753+338$ & 0.619 & 0.578 \\
$1759+753$ & 0.366 & 0.396 \\
$1810+522$ & 0.360 & 0.429 \\
$1811+317$ & 0.241 & 0.224 \\
$1818+551$ & 0.954 & 1.006 \\
$1838+575$ & 1.227 & 1.323 \\
$2052+239$ & 1.146 & 1.326 \\
$2111+801$ & 0.412 & 0.386 \\
$2128+333$ & 0.586 & 0.576 \\
$2247+381$ & 0.420 & 0.376 \\
$2316+238$ & 0.730 & 1.077 \\
$2322+396$ & 0.860 & 0.831 \\
\hline & & \\
\hline & & \\
135 &
\end{tabular}

Notes. The first column lists the name of the targets, the second and third columns list the formal uncertainties of the simulated white-phase noise added to the initial positions (one formal uncertainty was randomly affected for one quasar).

will be used for the link with the ICRF. This is still a large number compared to the overall amount of currently known targets that are suitable for the link between the reference frames (117 targets have been published by Bourda et al. 2008, 2011) or compared to the number of targets studied in the frame of the present work ( $40 \%$ of the previous targets). 
Table 6. Determination of the transformation parameters.

\begin{tabular}{ccc}
\hline \hline Differences (mas) & $\Delta \alpha \cos \delta$ & $\Delta \delta$ \\
\hline Average & 0.001 & 0.029 \\
Weighted rms & 0.899 & 0.850
\end{tabular}

Rotation, dipole ( $\mu$ as)

$\begin{array}{lc}\text { R1 } & 94.04 \pm 71.3 \\ \text { R2 } & 82.05 \pm 64.8 \\ \text { R3 } & -5.71 \pm 30.8 \\ \text { D1 } & 97.96 \pm 58.6 \\ \text { D2 } & 158.50 \pm 61.4 \\ \text { D3 } & 14.39 \pm 39.9\end{array}$

Rotation only ( $\mu$ as)

$\begin{array}{ll}\text { R1 } & 44.55 \pm 28.6 \\ \text { R2 } & 21.41 \pm 21.6 \\ \text { R3 } & -6.49 \pm 29.3\end{array}$

Notes. The first part of the table lists the statistics for the coordinate comparison. The second part presents the rotation angles and dipole parameters, together with their associated precision (standard deviation of the mean). The last part lists the rotation parameters alone, together with their associated precision, as previously. 3760 observations were used (80 observations of 47 targets during the five years of the Gaia mission).

It is beyond the scope of this paper to undertake a detailed study of the precision of the link due to the variability of quasars. The results above are therefore very preliminary and only valid for the limited set of targets studied in this paper. Nevertheless, they show that the quasar variability has a non-negligible effect on the precision of the link between the reference frames. A more sophisticated analysis is currently developed and will be published very soon.

\section{Conclusion}

We have presented, for the first time, optical observations of 47 targets that are suitable for the link between the ICRF and the future Gaia-CRF. They were observed, sometimes at the same time as Gaia, with a network of nine telescopes. This allowed us to compare ground-based observations to the Gaia magnitudes. For $95 \%$ of the sources, these observations bring new information about their magnitude variability. Notes about their morphology, retrieved from the CDS, show that $75 \%$ have an unknown morphology.

We also presented the Allan time variance, a statistical tool that could help to quantify the optical stability of a target (QSOs in the framework of this paper, but, more generally, any type of variable object). It could be of general use to find new suitable targets for the link between the reference frames if an astrophotometric relation could be confirmed (Popovic et al. 2012; Shabala et al. 2014). It must also be noted that Allan variances are used to quantify the astrometric stability of the RA/DEC time series of the ICRF targets (Gattano, 2017). Allan variances also provide an estimation of the longest averaging period, $P_{\max }$, in a set of observed magnitudes, during which it is possible to average the data. For a period shorter than $P_{\max }$, the underlying statistical process is stationary. In other words, the magnitude measurement is only affected by the measurement noise. Height targets, which are particularly well observed by the TAROT tele- scopes during more than five years, were used to determine $P_{\max }$ for each source. We showed that these periods are between $20 \mathrm{~d}$ and $70 \mathrm{~d}$. In the case of Gaia, several intermediate magnitudes are averaged to provide a single value in the catalog. Generally speaking, Gaia observes a target during a period longer than $P_{\max }$. As a consequence, the Gaia magnitudes provided in the catalog may not be affected by the measurement noise alone (for example, periodic astrophysical processes can affect the numerical value of the average magnitude). It would therefore be very important to have access to the intermediate magnitudes for each target observed by Gaia so that we can determine $P_{\max }$ in each case, to be able to conclude about the averaging process. Another interest in having access to the intermediate magnitudes would be to schedule observations in other wavelengths, such as radio, with the Very Long Baseline Array (VLBA) facilities. Multiwavelength observations of quasars are of interest for astrophysicists. Moreover, intermediate time-series of astrometry could be very interesting for determining astro-photometric correlations in the data and for studying the $P_{\max }$ averaging period for these astrometric time series. This last issue, together with the intermediate magnitudes, needs to be addressed in the Test Case WP944-VAL-060-005 of the Core-Unit 9 inside the Gaia-DPAC. This work is not for scientific validation purposes, but to provide good-quality data to the astronomical community beyond the Gaia community.

Finally, we investigated the consequences of quasar variability on the precision of the link between reference systems. Preliminary computations showed that if the coordinates of the targets studied in this paper were affected by a white-phase noise with a formal uncertainty of about 1 mas (due to astrophysical processes that are put in evidence by the magnitude variations of the sources), it would have an effect on the precision of the link at the level of $50 \mu$ as. Taking into account that this result has been obtained with a simplified model, the level of precision is, nevertheless, more than ten times the precision of the link when quasar variability is not taken into account.

Quasars must be considered as very special objects in the framework of the Gaia mission because they are extended and variable objects. Their light curves might allow us to anticipate some morphological changes, which is of importance for astrophysicists and astrometrists both.

Acknowledgements. G. Damljanovic acknowledges the observing grant support from the Institute of Astronomy and Rozhen National Astronomical Observatory, Bulgarian Academy of Sciences, through the bilateral joint research project "Observations of ICRF radio-sources visible in optical domain" (head G. Damljanovic). This work is a part of the project No. 176011 ("Dynamics and kinematics of celestial bodies and systems") supported by the Ministry of Education, Science and Technological Development of the Republic of Serbia. This work has made use of data from the European Space Agency (ESA) mission Gaia (https : //www . cosmos.esa.int/gaia), processed by the Gaia Data Processing and Analysis Consortium (DPAC; https://www.cosmos.esa.int/web/ gaia/dpac/consortium). Funding for the DPAC has been provided by national institutions, in particular, the institutions participating in the Gaia Multilateral Agreement.

\section{References}

Abazajian, K., Adelman, J., Agueros, M., et al. 2009, ApJS, 182, 543 Andrei, A., Bouquillon, S., de Camargo, J., et al. 2008, in JSRS and X. Lohrmann Kolloquium Proc., eds. M. Soffel, \& N. Capitaine, 199

Bahcall, J., Kirhakos, S., \& Schneider, D. 1995, ApJ, 452, L91 Bouquillon, S., Barache, C., Carlucci, T., et al. 2014, SPIE, 9152, 915203 Bourda, G.,Charlot, P., \& Le Campion, J. 2008, A\&A, 490, 403 Bourda, G., Collioud, A., Charlot, P., et al. 2011, A\&A, 526, A102 Carrasco, J., Evans, D., Montegriffo, P., et al. 2016, A\&A, 595, A7 
Cheung, C., Wardle, J., \& Chen, T. 2003, New Astron. Rev., 47, 423 Chonis, T., \& Gaskell, C. 2008, AJ, 135, 264

Damljanovic, G., Vince, O., \& Boeva, S. 2014, Serb. Astron. J., 188, 85 ESA, DPAC 2016, Gaia Data Release 1, Documentation release 1.0

Eyer, L., \& Mignard, F. 2005, MNRAS, 361, 1136

Fabricius, C., Bastian, U., Portell, J., et al. 2016, A\&A, 595, A3

Falomo, R., Bettoni, D., Karhunen, J., et al. 2014, MNRAS, 440, 476

Gaia Collaboration (Prusti, T., et al.) 2016a, A\&A, 595, A1

Gaia Collaboration (Brown, A., et al.) 2016b, A\&A, 595, A2

Gattano, C. 2016, Ph.D., Thèse de doctorat de l'Université Paris Sciences et lettres

Hawkins, M. 2003, MNRAS, 344, 492

Honeycutt, R. 1992, PASP, 104, 435

IEEE 1999, IEEE Std 11399

Jordi, C., Gebran, M., Carrasco, J., et al. 2010, A\&A, 523, A48

Kacprzak, G., Churchill, C., Evans, J., et al. 2011, MNRAS, 416, 3118

Kelly, B., Bechtold, J., \& Siemiginowska, A. 2009, ApJ, 698, 895

Kovalev, Y., Petrov, L., \& Plavin, A. 2017, A\&A, 598, L1

Lambert, S. 2013, A\&A, 553, A122

Li, S., \& Cao, X. 2008, MNRAS, 387, L41

Lindegren, L., Lammers, U., Bastian, U., et al. 2016, A\&A, 595, A4

Lobanov, A., \& Roland, J. 2005, A\&A, 431, 831

Mary, D., Kurtz, D., Martinez, P., et al. 2006, Gaia document Gaia-C7-TN-ARIDM-003-1

Mignard, F. 2012, Mem. Soc. Astron. It., 918, 83

Mignard, F. 2013, IMCCE International Workshop NAROO-GAIA A new reduction of old observations in the Gaia era, Paris observatory, France
Mignard, F., \& Klioner, S. 2012, A\&A, 547, A59

Mignard, F., Klioner, S., Lindegren, L., et al. 2016, A\&A, 595, A5

Newberg, H., \& Yanni, B. 1997, ApJS, 113, 89

Nilsson, K., Pursimo, T., Heidt, J., et al. 2003, A\&A, 400, 95

Nilsson, K., Pasanen, M., Takalo, L., et al. 2007, A\&A, 475, 199

Palanque-Delabrouille, N., Yeche, Ch., Myers, A., et al. 2011, A\&A, 530, A122

Paturel, G., Petit, C., Prugniel, Ph., et al. 2003, A\&A, 412, 45

Petrov, L. 2011, AJ, 142, 105

Petrov, L., \& Kovalev, L. 2017, MNRAS, 467, L71

Popovic, L., Jovanovic, P., Stalevski, M., et al. 2012, A\&A, 538, A107

Ravi, J., \& Hum, C. 2013, MNRAS, 429, 1717

Riley, W. 2008, NIST special publication 1065, Handbook of frequency stability analysis

Shabala, S., Rogers, J., McCallum, J., et al. 2014, J. Geod., 88, 575

Shalyapin, V., Goicoetchea, L., \& Alcalde, D. 2002, ApJ, 579, 127

Sullivan, D., Allan, D., Howe, D., et al. 1990, NIST Technical Note, 1337

Taris, F., Souchay, J., Andrei, A., et al. 2011, A\&A, 526, A25

Taris, F., Andrei, A., Klotz, A., et al. 2013, A\&A, 552, A98

Taris, F., Andrei, A., Roland, J., et al. 2016, A\&A, 587, A112

Urry, C., Scarpa, R., O’Dowd, M., et al. 2000, ApJ, 532, 816

Varadi, M., Eyer, L., Jordan, S., et al. 2009, in Stellar Pulsation, Challenges for Theory and Observation, AIP, eds. J. Guzik \& P. Bradley

Wierzcholska, A., Ostrowski, M., Starwarz, L., et al. 2015, A\&A, 573, A69

Wu, X., Hao, G., Jia, Z., et al. 2012, AJ, 144, 49

York, D., Adelman, J., Anderson, J., et al. 2000, AJ, 120, 1579 


\section{Appendix A: Allan variances}

\section{A.1. Power spectral density and Allan variances}

Atomic clocks and frequency standards are affected by five types of noises: white-phase noise (White PM), flicker-phase noise (Flicker PM), random-walk-phase noise and white-frequency noise (RW PM/White FM), flicker-frequency noise (Flicker FM), and random-walk-frequency noise (RW FM). These noises are well known in the spectral domain, where they can be characterized by their power spectral density. For an oscillator such as an atomic clock, five types of noises can be found, and the power spectral density can be modeled by

$S_{y}(f)=\sum_{\alpha=-2}^{2} h_{\alpha} \cdot f^{\alpha}$,

where $h_{\alpha}$ are constants. One value of $\alpha$ corresponds to one type of noise (see Table A.1).

A random process is stationary when its statistical properties (mean and variance) do not vary in time. If $-1<\alpha<+1$, then the random process is stationary, and if $\alpha<-1$, it is a nonstationary process.

In the time domain, sequential average-frequency instabilities are defined by a two-sample deviation, also called the Allan deviation (IEEE 1999), which is the square root of a two-sample variance, also called the Allan variance (AVAR). This variance does not take dead time between frequency samples into account, all the measurements are assumed to be made continuously. Of course, it is not always possible to fulfill this requirement, and this generates jumps in the time series owing to non-periodic measurements or to the lack of some of them. There is no simple solution to this problem. If the Allan variance must be calculated for an averaging period $\tau$ much longer than $\tau_{0}\left(\tau_{0}\right.$ being the time interval between two consecutive measurements), it is permitted to spread the measurements following an average periodicity $\tau_{0}^{\prime}$ computed as the average of all the time intervals between two consecutive measurements.

For a sampling interval $\tau$

$\sigma_{y}^{2}(\tau)=\frac{1}{2}\left\langle\left[\bar{y}_{k+1}-\bar{y}_{k}\right]^{2}\right\rangle$,

where

$\bar{y}_{k}=\frac{x_{k+1}-x_{k}}{\tau}$.

The brackets denote an infinite time average, and $\tau$ is the sampling interval. In practice, the requirement of an infinite time average is never fulfilled, and the Allan deviation is estimated by

$\sigma_{y}(\tau)=\left[\frac{1}{2(M-1)} \sum_{k=1}^{M-1}\left(\bar{y}_{k+1}-\bar{y}_{k}\right)^{2}\right]^{1 / 2}$,

where $M$ is the number of frequency measurements. The Allan deviation can also be expressed in terms of time-difference (or time-residual) measurements by combining the two last equations,

$\sigma_{y}(\tau)=\left[\frac{1}{2(N-2) \tau^{2}} \sum_{k=1}^{N-2}\left(x_{k+2}-2 x_{k+1}+x_{k}\right)^{2}\right]^{1 / 2}$,

where $x_{k}, x_{k+1}$ and $x_{k+2}$ are time residual measurements made at $t_{k}, t_{k+1}=t_{k}+\tau$ and $t_{k+2}=t_{k}+2 \tau$, respectively. $k=1,2,3, \ldots$,
Table A.1. Characteristic slopes for $S_{y}(f), \operatorname{Mod} \sigma_{y}^{2}(\tau)$, and $\sigma_{x}^{2}(\tau)$ on a bilogarithmic diagram (or exponent of the power-law model).

\begin{tabular}{cccc}
\hline \hline$S_{y}(f)$ & $\operatorname{Mod} \sigma_{y}^{2}(\tau)$ & $\sigma_{x}^{2}(\tau)$ & Type of noise \\
\hline-2 & 1 & 3 & Random-walk FM \\
-1 & 0 & 2 & Flicker FM \\
0 & -1 & 1 & White FM - Random-walk PM \\
+1 & -2 & 0 & Flicker PM \\
+2 & -3 & -1 & White PM \\
\hline
\end{tabular}

$N$ is the number of time measurements, and $1 / \tau$ is the nominal fixed sampling rate that gives zero dead-time between frequency measurements.

When differentiating between white- and flicker-phase modulation noise is desirable, a modified deviation, denoted $\operatorname{Mod} \sigma_{y}(\tau)$, may be used to characterize frequency instabilities. This modified deviation is

$$
\begin{aligned}
\operatorname{Mod} \sigma_{y}(\tau)= & \left\{\frac{1}{2 \tau^{2} m^{2}(N-3 m+1)} \sum_{j=1}^{N-3 m+1}\right. \\
& \left.\times\left[\sum_{i=j}^{m+j-1}\left(x_{i+2 m}-2 x_{i+m}+x_{i}\right)\right]^{2}\right\}^{1 / 2} .
\end{aligned}
$$

$m=2^{p}$ with $p=0,1,2,3 \ldots$ Unlike $\sigma_{y}(\tau), \operatorname{Mod} \sigma_{y}(\tau)$ has the property of yielding different dependencies on $\tau$ for white-noise and flicker-phase noise. The dependencies are $\tau^{-3 / 2}$ and $\tau^{-1}$, respectively. The square of $\operatorname{Mod} \sigma_{y}(\tau)$ is often denoted MVAR (modified Allan variance).

It can be demonstrated that the relation between the power spectral density and MVAR is

$\operatorname{Mod} \sigma_{y}^{2}(\tau)=\frac{2}{(m \pi \tau)^{2}} \int_{0}^{f_{h}} S_{y}(f) \frac{\sin ^{6}(f \pi \tau)}{f^{2} \sin ^{2}\left(f \pi \tau_{0}\right)} \mathrm{d} f$,

with $\tau=m \tau_{0}$ and $f_{h}$ a high-frequency cutoff of an infinitely sharp low-pass filter. This last equation can be expressed as a power law of $\tau$. On a bilogarithmic plot, the five different types of noise are then represented by straight lines with characteristic slopes (Fig. A.1). Table A.1 gives the link between the characteristic slopes in the time (MVAR, TVAR) and frequency $\left(S_{y}(f)\right)$ domains.

Finally, the time Allan variance, TVAR, with square root TDEV, is a measure of time stability based on the modified Allan variance. It is defined as

$\sigma_{x}^{2}(\tau)=\frac{\tau^{2}}{3} \operatorname{Mod} \sigma_{y}^{2}(\tau)$

The time Allan variance is equal to the standard variance of the time deviations for white-phase modulation noise.

\section{A.2. Effect of a periodic signal on the Allan variances}

A periodic signal has a specific signature in terms of Allan variances. It can be demonstrated that the representative curve is affected by an oscillation, the maxima of which are located at averaging times:

$\tau=(2 k+1) \frac{T_{0}}{2}$.

In a bilogarithmic plot, if the signal is greater than the noise, a periodic signal of period $T_{0}$ will be seen as a succession of 
A\&A 611, A52 (2018)

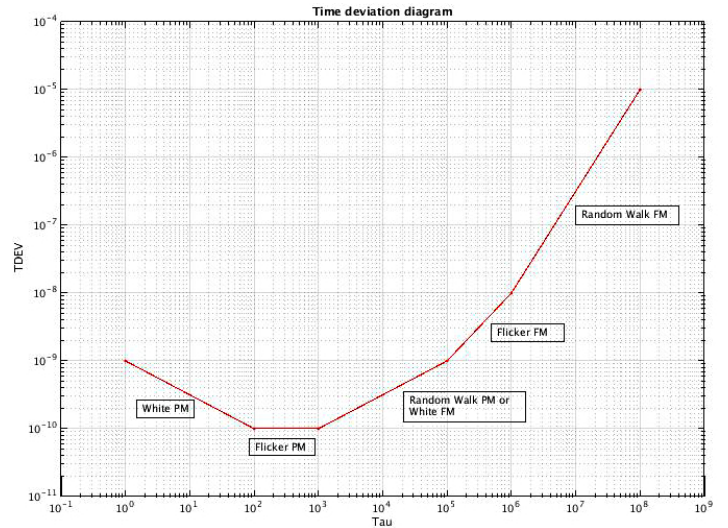

Fig. A.1. TDEV of the five types of noise with their characteristic slopes.

bumps whose tops are located at averaging times equal to odd multiples of the half-period. Figure A. 2 shows the time Allan deviation of a simulated white-phase noise affected by a periodic signal.

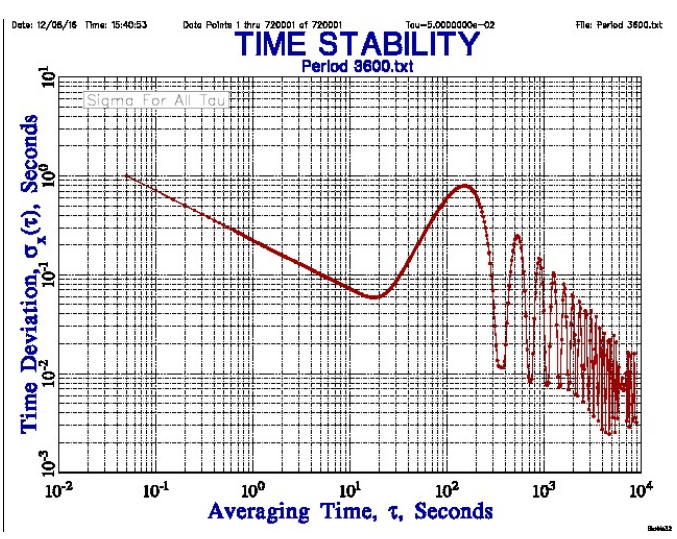

Fig. A.2. TDEV of a white-phase noise affected by a periodic signal of period $T_{0}=360 \mathrm{~s}$. The maxima are visible for averaging times $\tau=$ $\frac{T_{0}}{2}, \frac{3 T_{0}}{2}, \frac{5 T_{0}}{2}, \ldots$ 


\section{Appendix B: Additional figures}
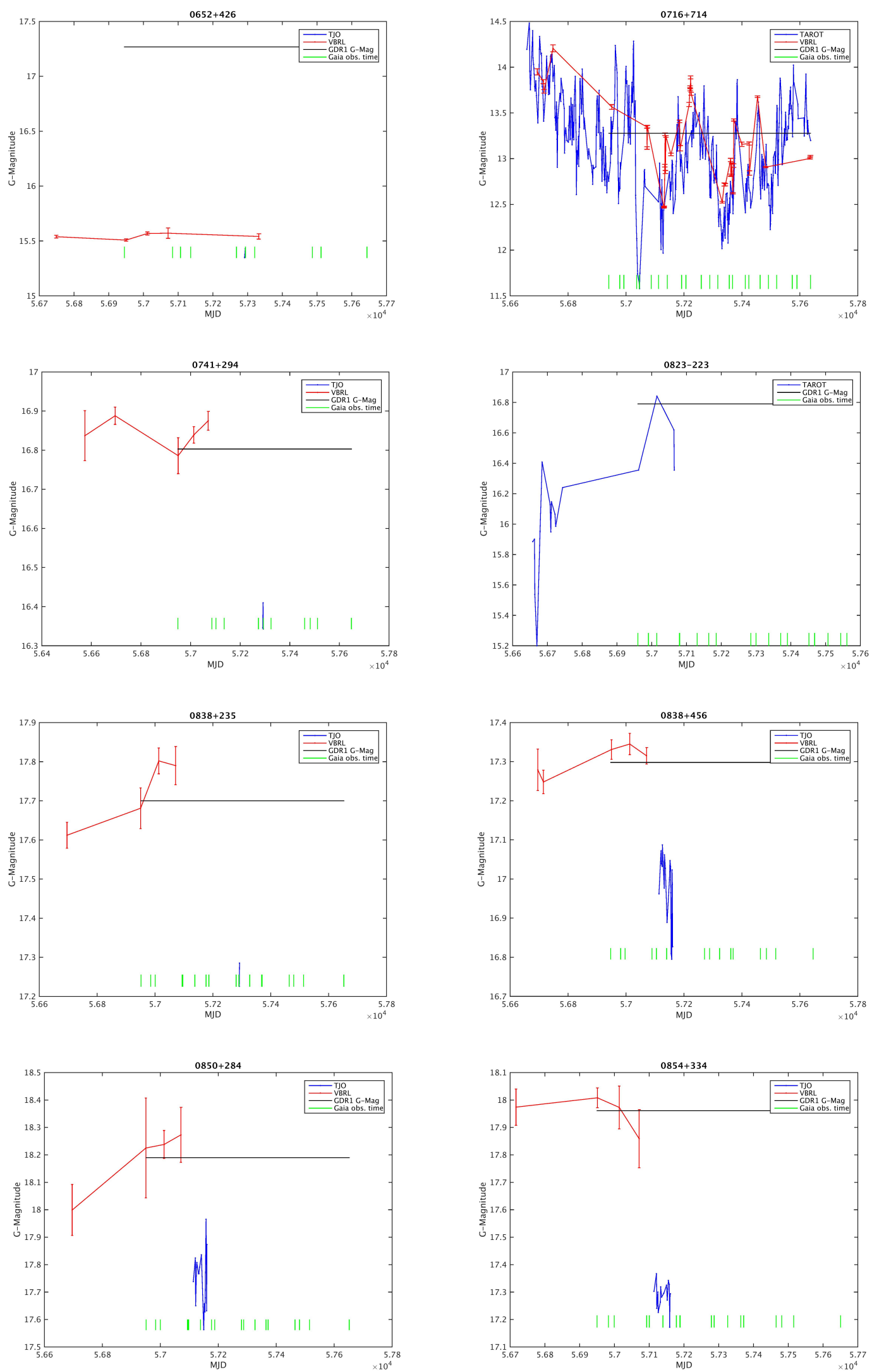

Fig. B.1. Comparison of $G$ magnitudes for QSOs 0652+426, 0716+714, 0741+294, 0823-223, 0838+235, 0838+456, 0850+284, and 0854+334 from top to bottom and left to right. The vertical green ticks are the observation times by Gaia, the horizontal black line is the GDR1 magnitude. The other curves are the $G$ magnitudes observed by the Telescope Joan Oro (TJO), the TAROT telescopes (TAROT), and/or the VidojevicaBelogradchik-Rozhen-LFOA telescopes (VBRL), as indicated at the top right of each panel. 

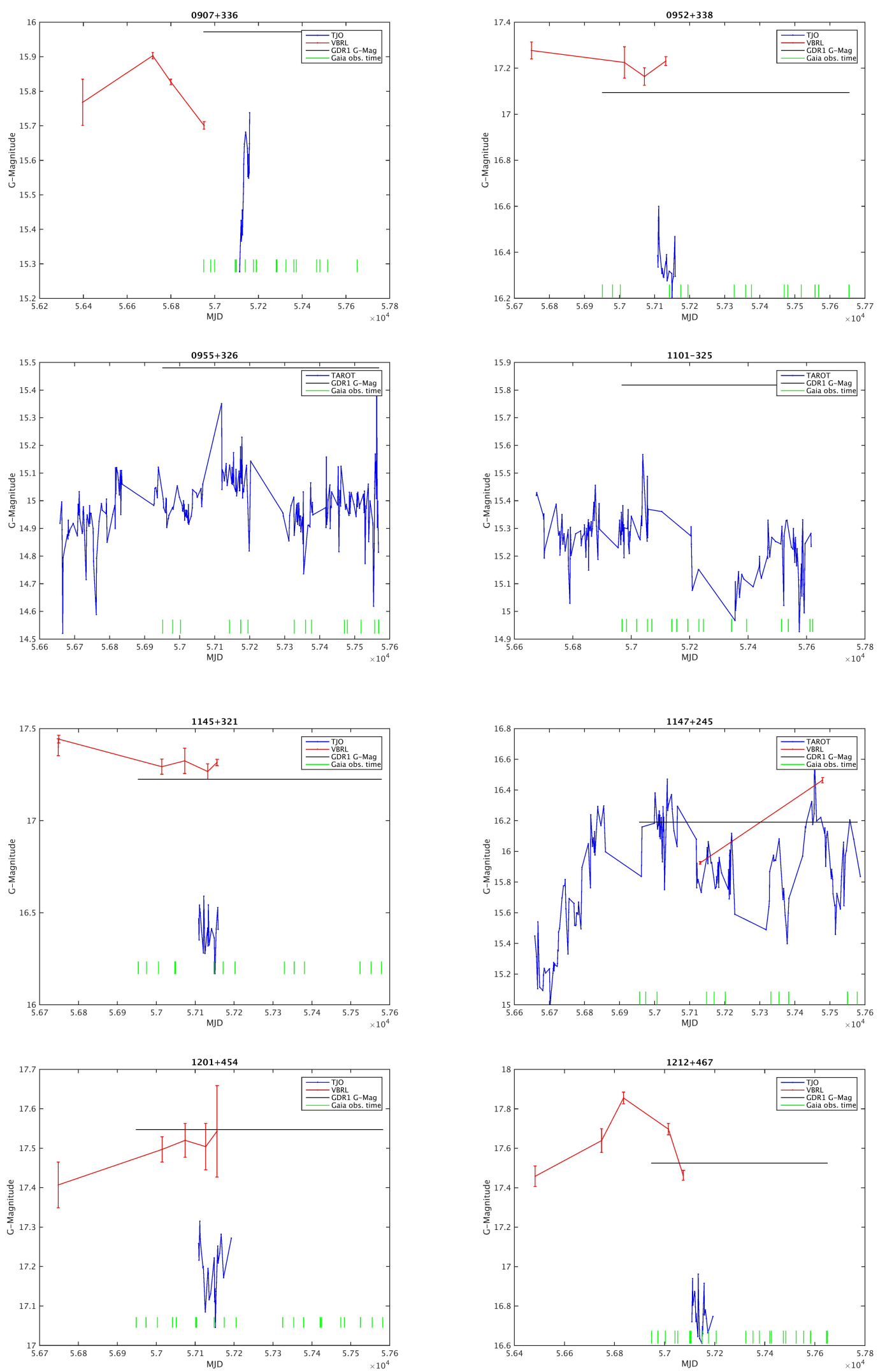

Fig. B.2. Comparison of $G$ magnitudes for QSOs 0907+336, 0952+338, 0955+326, 1101-325, 1145+321, 1147+245, 1201+454, and 1212+467 from top to bottom and left to right. The vertical green ticks are the observation times by Gaia, the horizontal black line is the GDR1 magnitude. The other curves are the $G$ magnitudes observed by the Telescope Joan Oro (TJO), the TAROT telescopes (TAROT), and/or the VidojevicaBelogradchik-Rozhen-LFOA telescopes (VBRL), as indicated at the top right of each panel. 

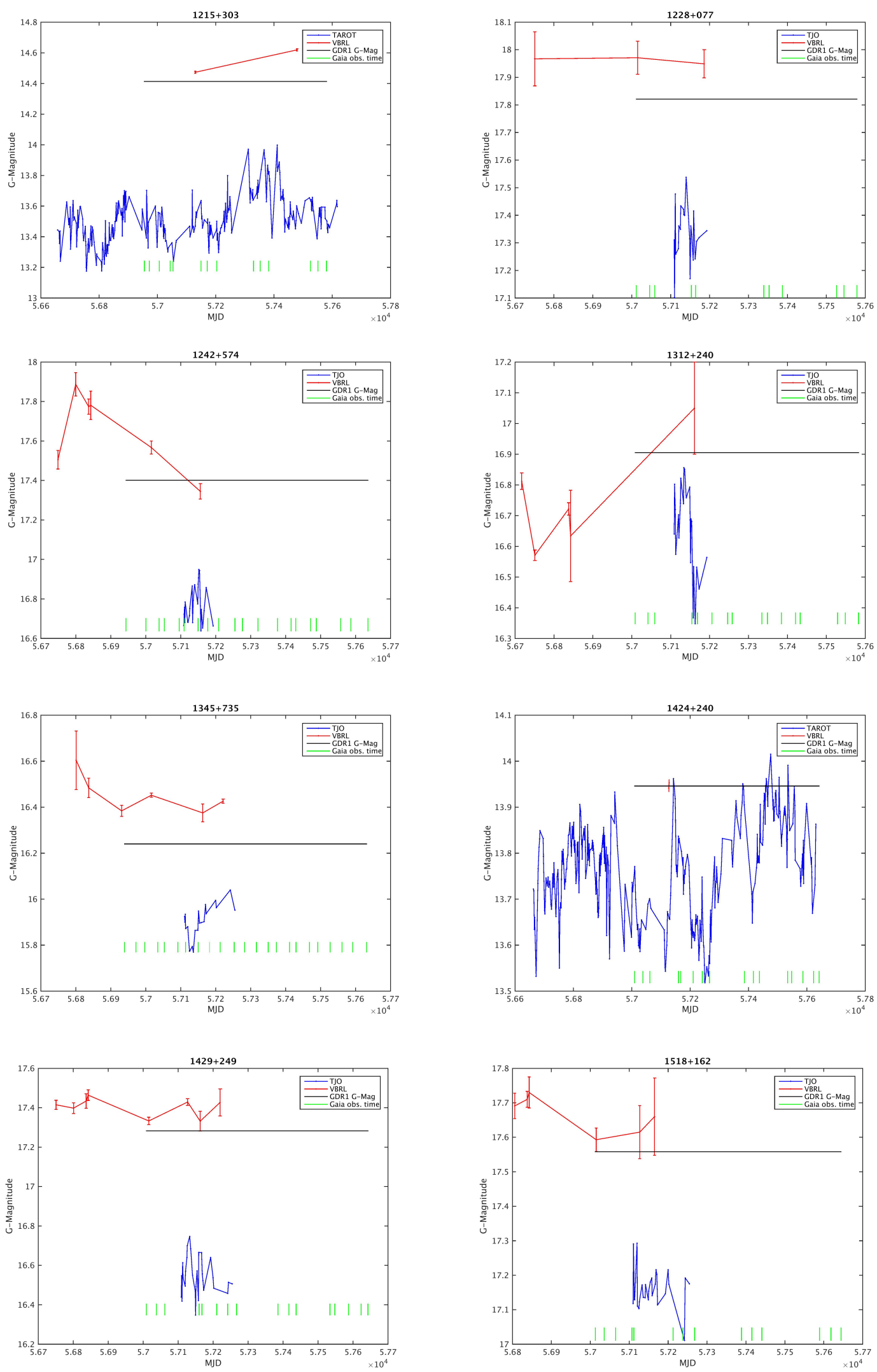

Fig. B.3. Comparison of $G$ magnitudes for QSOs $1215+303,1228+077,1242+574,1312+240,1345+735,1424+240,1429+249$, and $1518+162$ from top to bottom and left to right. The vertical green ticks are the observation times by Gaia, the horizontal black line is the GDR1 magnitude. The other curves are the $G$ magnitudes observed by the Telescope Joan Oro (TJO), the TAROT telescopes (TAROT), and/or the VidojevicaBelogradchik-Rozhen-LFOA telescopes (VBRL), as indicated at the top right of each panel. 

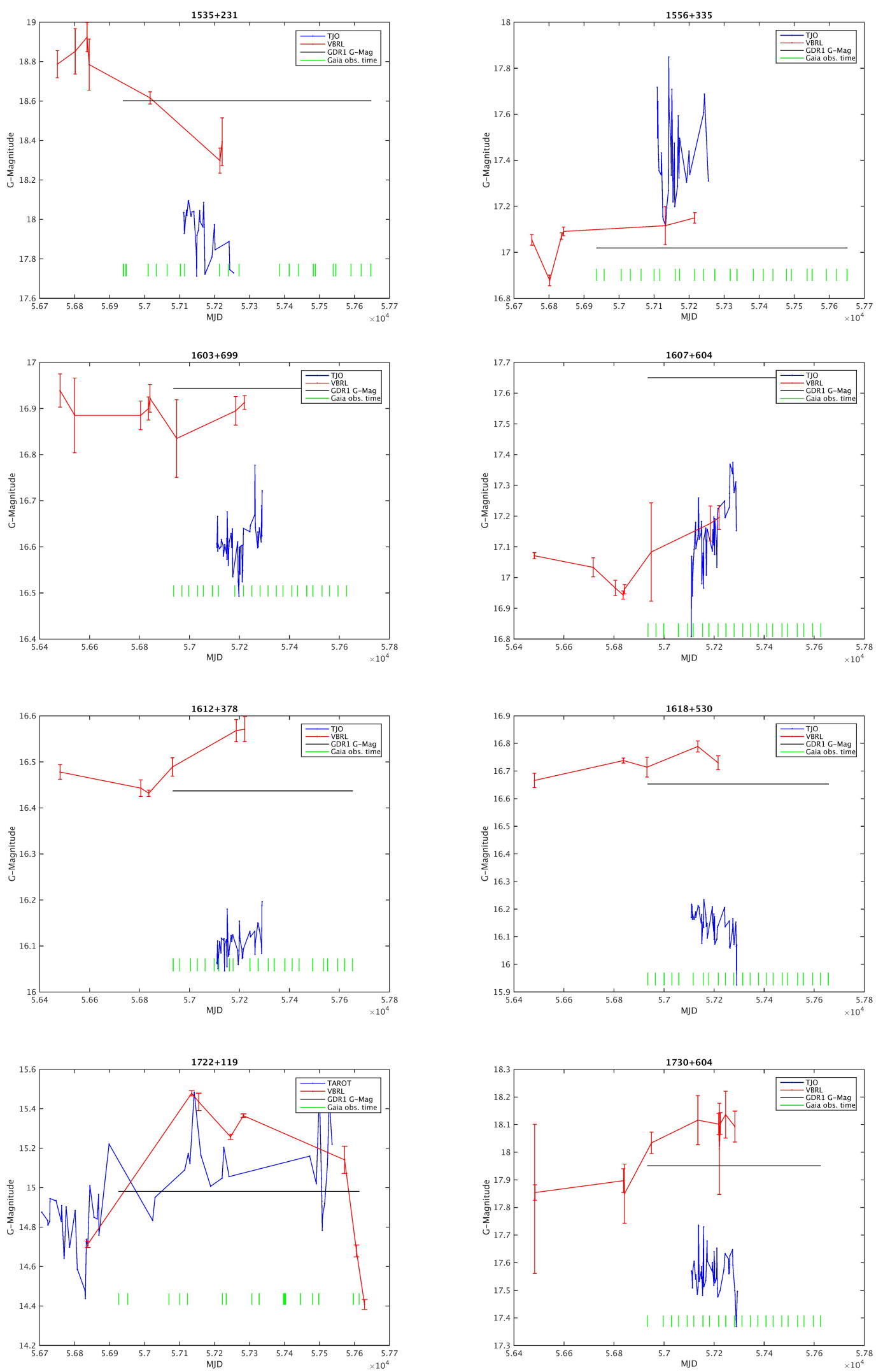

Fig. B.4. Comparison of $G$ magnitudes for QSOs $1535+231,1556+335,1603+699,1607+604,1612+378,1618+530,1722+119$, and 1730+604 from top to bottom and left to right. The vertical green ticks are the observation times by Gaia, the horizontal black line is the GDR1 magnitude. The other curves are the $G$ magnitudes observed by the Telescope Joan Oro (TJO), the TAROT telescopes (TAROT), and/or the VidojevicaBelogradchik-Rozhen-LFOA telescopes (VBRL), as indicated at the top right of each panel. 

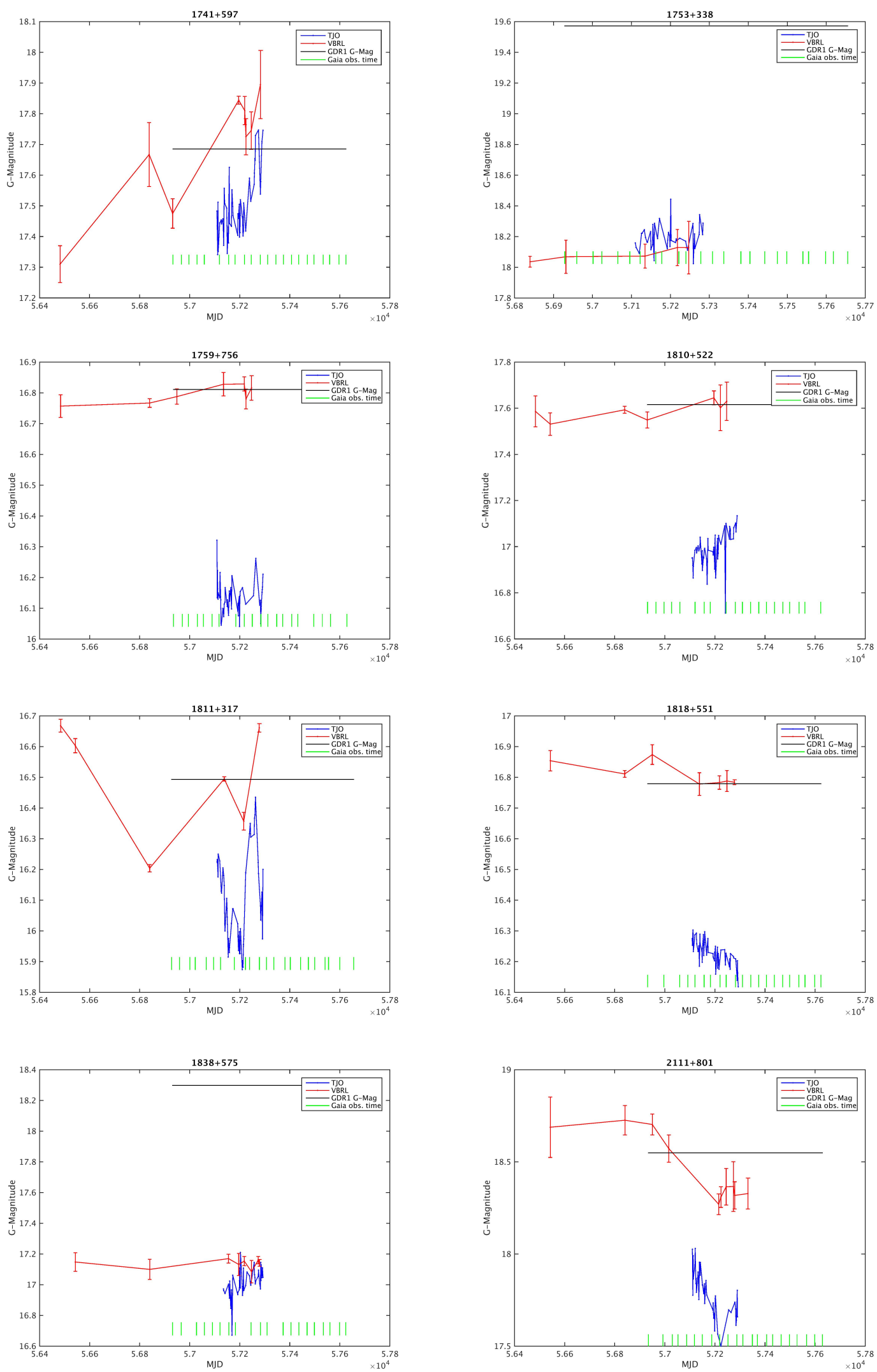

Fig. B.5. Comparison of $G$ magnitudes for QSOs $1741+597,1753+338,1759+756,1810+522,1811+317,1818+551,1838+575$, and $2111+801$ from top to bottom and left to right. The vertical green ticks are the observation times by Gaia, the horizontal black line is the GDR1 magnitude. The other curves are the $G$ magnitudes observed by the Telescope Joan Oro (TJO), the TAROT telescopes (TAROT), and/or the VidojevicaBelogradchik-Rozhen-LFOA telescopes (VBRL), as indicated at the top right of each panel. 

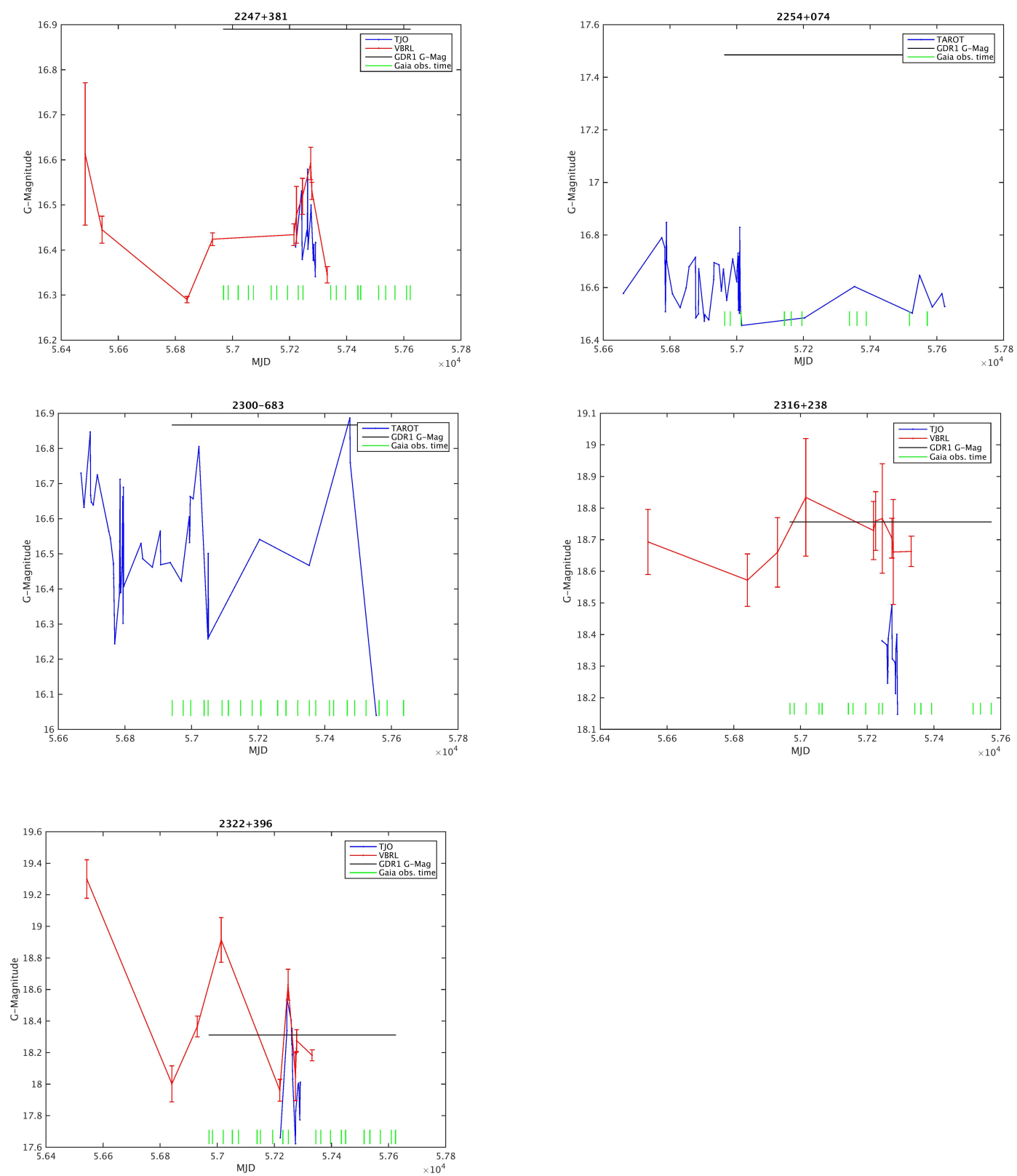

Fig. B.6. Comparison of $G$ magnitudes for QSOs $2247+381,2254+074,2300-683$, 2316+238, and 2322+396 from top to bottom and left to right. The vertical green ticks are the observation times by Gaia, the horizontal black line is the GDR1 magnitude. The other curves are the $G$ magnitudes observed by the Telescope Joan Oro (TJO), the TAROT telescopes (TAROT), and/or the Vidojevica-Belogradchik-Rozhen-LFOA telescopes (VBRL), as indicated at the top right of each panel. 